\title{
Hierarchical Distributed Control Strategy for Electric Vehicle Mobile Energy Storage Clusters
}

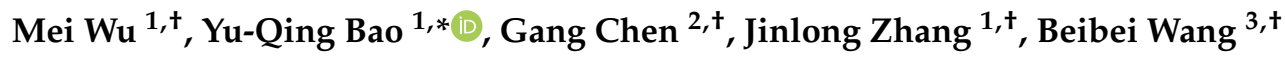 \\ and Weixing Qian ${ }^{1,+}$ \\ 1 NARI School of Electrical Engineering and Automation, Nanjing Normal University, Nanjing 210023, China; \\ wumeijq@hotmail.com (M.W.); ZJL0310@163.com (J.Z.); 61192@njnu.edu.cn (W.Q.) \\ 2 State Grid Suqian Power Supply Company, Suqian 223800, China; ywy_cheng@163.com \\ 3 School of Electrical Engineering, Southeast University, Nanjing 210096, China; wangbeibei@seu.edu.cn \\ * Correspondence: baoyuqing@nju.edu.cn; Tel.: +158-6181-3535; Fax: +86-25-8548-1270 \\ + These authors contributed equally to this work.
}

Received: 6 March 2019; Accepted: 25 March 2019; Published: 27 March 2019

check for updates

\begin{abstract}
The stability problem of the power system becomes increasingly important for the penetration of renewable energy resources (RESs). The inclusion of electric vehicles (EVs) in a power system can not only promote the consumption of RESs, but also provide energy for the power grid if necessary. As a mobile energy storage unit (MESU), EVs should pay more attention to the service life of their batteries during operation. A hierarchical distributed control strategy was proposed in this paper for mobile energy storage clusters (MESCs) considering the life loss of each EV's battery. This strategy was divided into a two-layer control structure. Firstly, numerous EVs were divided into different clusters according to their regional relationships. The lower layer adopted a distributed collaborative control approach for allocating energy among EVs in the cluster. Under this condition, an aggregate EVs response model was established and the characteristic of the MESC was analyzed. Secondly, the upper layer applied the multi-agent consensus algorithm to achieve the optimal allocation among different clusters. Therefore, the control strategy realized the two-way communication of energy between EVs and the power grid, and ensured the optimal economical dispatch for the mobile energy storage system (MESS). Finally, the simulation of testing examples verified the effectiveness of the proposed strategy.
\end{abstract}

Keywords: mobile energy storage system (MESS); multi-agent; consensus algorithm

\section{Introduction}

Faced with an energy crisis and serious environmental problems, countries are developing renewable energy technologies in order to meet their domestic energy demand and strive for sustainable development. However, the random and intermittent characteristics of renewable energy sources (RESs) impact the power system reliability [1,2].

The introduction of energy storage devices effectively solves the problem of grid-connected renewable energy generation [3,4]. However, the high investment and construction costs of energy storage devices will increase the cost of the energy storage system (ESS). The application of electric vehicles (EVs) as mobile energy storage units (MESUs) has drawn widespread attention under this circumstance [5,6]. A large amount of EVs are connected to the power grid, which is equivalent to controllable loads or the mobile energy storage cluster (MESC) that supports ancillary services. By controlling the charging/discharging of EVs' batteries, it could not only promote the consumption of RESs, but also be beneficial to peak-load shifting and valley-filling [7,8]. Moreover, large amounts of EVs can contribute to voltage regulation and frequency regulation when considering the characteristics of energy storage [9]. According to the concept of vehicle-to-grid (V2G), the energy stored in the 
batteries of EVs can also be sent back to the power grid when there is a shortage of active power [10]. Since an EV is a carrier of batteries, the control strategy designed to allocate energy among EVs is equivalent to the control method of an energy storage unit (ESU). Due to the unique advantages of EVs in terms of their demand response (DR) and energy storage, the rational dispatch of energy in the mobile energy storage system (MESS) will be an inevitable requirement for the development of smart grids [11].

In terms of regulation control of ESUs, a past study [12] established a joint scheduling model of a wind power storage hybrid system on multiple time scales and gave the production plan of the hybrid system the day before and during that day. Another study [13] used a small optical storage joint system, proposing a real-time economic dispatch method. In Hong et al.'s study [14] a method was proposed for the optimal control of a battery's ESS based on a model predictive control for a real-time power fluctuation of a wind farm. However, the above studies all adopted the conventional centralized control to solve the dispatch problem of the ESS. The corresponding ESS also was distributed because of the decentralized distribution of RESs, and the scale of the ESS will continue to expand with the continuous penetration of RESs. The conventional centralized control method may encounter severe challenges. In order to achieve the optimal operating conditions, a large number of scheduling commands and device feedback information will easily impose a substantial computational burden, causing control center communication congestion or even dimensionalitycurse.

Compared with the conventional centralized control, the distributed control can realize the sharing of global information in the system only by relying on the information interaction between adjacent devices [15]. Applying the distributed control to the ESS can mitigate the dependence on the control center and improve the robustness and flexibility of the system [16,17].

Due to the high cost of energy storage devices, not only the accurate release of the scheduling commands, but also the life loss of the ESS need to be considered in distributed scheduling. In Chen et al. [18], a distributed cooperative control strategy for the MESU that considered the life loss cost of the EV's battery was proposed, which could not only realize the optimal allocation of energy among MESUs, but also guarantee minimum life loss cost of batteries. However, the scale of the MESS considered in this paper [18] was relatively small and easily caused a dimensionality curse in the face of numerous MESUs.

To fill this gap, this paper proposes a hierarchical distributed control strategy for determining the optimal allocation of energy among MESCs. By using this method, the minimum life loss cost of the MESS can be obtained in addition to meeting the demand of the power system.

The rest of the paper is as follows: the hierarchical distributed control method is presented in Section 2, the overall mathematical formulation of the proposed control strategy is described in detail in Section 3, simulation results are shown and discussed in Section 4 in order to verify the proposed method and finally, the paper is concluded in Section 5.

\section{Hierarchical Distributed Control}

Chen et al. [18] proposed a distributed cooperative control strategy for MESUs that considered the life loss cost. The ratio of the initial investment cost of the EV's battery to the cycle life is defined as the life loss cost of the EV's battery. The goal is to minimize the life loss cost and realize the optimal allocation of energy. On this basis, this paper proposes a hierarchical distributed control strategy with a two-layer control structure. The lower layer establishes the corresponding aggregated model according to the different energy allocation methods for batteries of EVs, and designs the distributed control strategy. Using the distributed control strategy in Chen et al. [18], the optimal allocation of energy was realized based on different response models in the lower layer.

The structure of the hierarchical distributed control is shown in Figure 1, which is divided into two layers that include the upper layer and the lower layer. Before the scheduling command is issued, the aggregated model of the MESC in the upper layer is modeled according to the parameters of the batteries in the lower layer. When the scheduling command begins to be issued, the control center 
sends the energy information to the leader node in the upper layer. The consensus algorithm is run after the leader node receives the information. Energy is allocated in the upper layer while ensuring the minimum total scheduling cost of the system. As soon as the allocation of energy in the upper layer is finished, all MESCs will serve as the control centers of the lower layer in order to transmit the energy information to the leader node of the lower layer. The lower layer can perform the energy allocation among EVs.

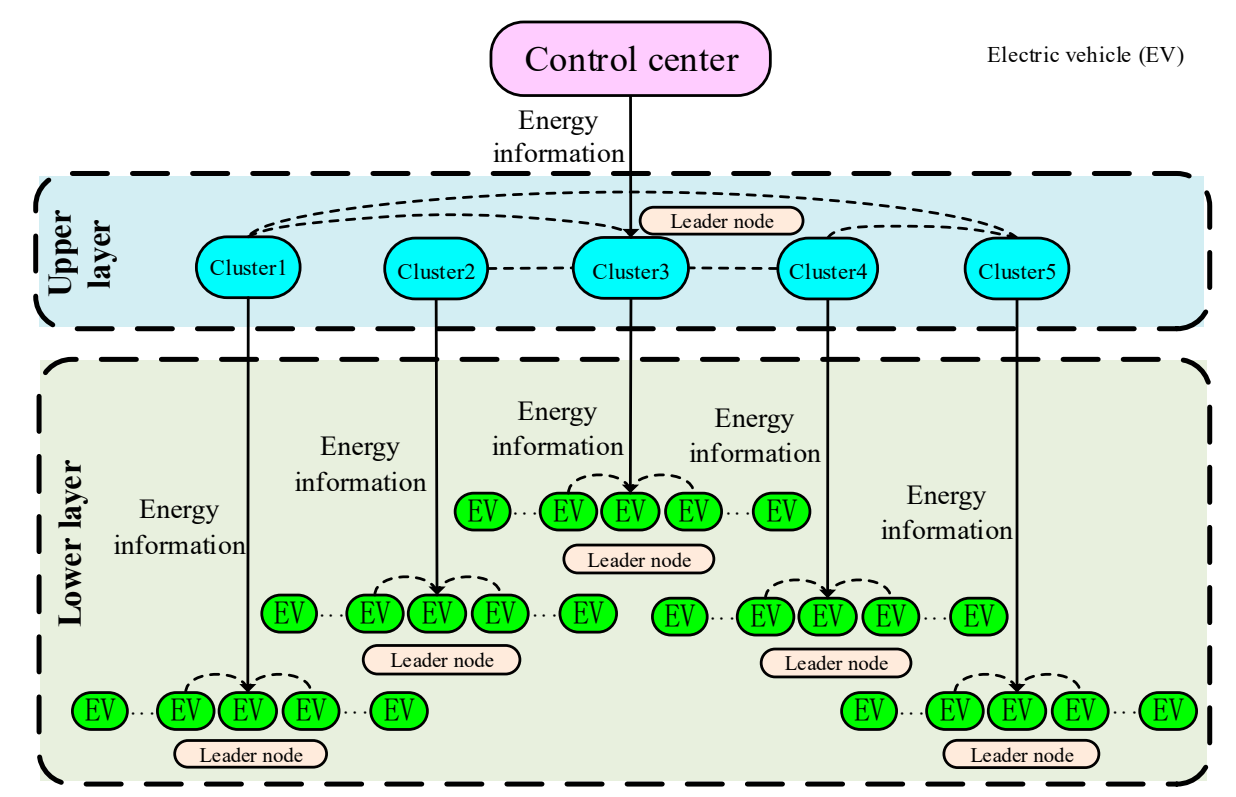

Figure 1. Communication topology of the mobile energy storage system (MESS).

\section{MESC Model}

\subsection{Lower Layer Control Strategy}

Considering the life loss cost of the EV's battery, the lower layer adopts the distributed control strategy based on the multi-agent consensus algorithm, where it regards numerous EVs as a multi-agent system. The optimal energy allocation among EVs is realized by the information interaction between the charging piles.

According to Chen et al. [18], the relationship between the life loss cost of the Li-ion battery and output energy is described by:

$$
F_{v}=\frac{C_{v}}{a_{v} Q_{\mathrm{N}, v}^{b_{v}}} \cdot Q_{v}^{b_{v}}
$$

where $v$ is the index of the $\mathrm{EV}, F_{v}$ denotes the life loss cost of the $\mathrm{EV} v, Q_{\mathrm{N}, v}$ denotes the rated capacity of the EV $v, Q_{v}$ is the amount of output energy released by the EV $v, C_{v}$ is the initial investment cost of the battery of the EV $v \cdot a_{v}$ and $b_{v}$ are coefficients.

As seen by Equation (1), during the discharge process, the smaller the amount of the power discharged, the smaller the life loss cost. Therefore, the optimal economical dispatch can be realized by allocating the energy of batteries reasonably.

The objective of the dispatch problem is to minimize the total cost of overall MESUs, which can be formulated as below:

$$
\min \quad F_{\mathrm{agg}}=\sum_{v=1}^{n} F_{v}\left(Q_{v}\right)
$$

where $n$ is the total number of EVs in the cluster and $F_{\text {agg }}$ denotes the total life loss cost.

When EVs are connected to the power grid, the initial energy of each battery is related to the driving distance, and the maximum energy is set by the owner. 
The optimization problem is subjected to the output energy limit constraint and energy balance constraint:

$$
\begin{gathered}
Q_{v, \min } \leq Q_{v} \leq Q_{v, \max } \\
\sum_{v=1}^{n} Q_{v}-Q_{\mathrm{D}}=0
\end{gathered}
$$

where $Q_{v, \text { max }}$ and $Q_{v, \text { min }}$ are the maximum and minimum output energy, respectively, that each EV can provide and $Q_{\mathrm{D}}$ denotes the total demand of the system.

In the distributed consensus algorithm, the incremental $\operatorname{cost} \lambda_{v}$ is the same as $I_{\mathrm{F}, v}$, where $I_{\mathrm{F}, v}$ can be obtained by calculating the partial derivative of the life loss $\operatorname{cost} F_{v}$ with respect to the output energy $Q_{v}$, which is represented as below:

$$
I_{\mathrm{F}, v}=\frac{\partial F_{v}\left(Q_{v}\right)}{\partial Q_{v}}=\lambda_{v} \quad i=1,2, \ldots, n
$$

The incremental $\operatorname{cost} \lambda_{v}$ is considered as the consensus variable. According to Equations (1) and (5), the output energy offered by each EV can be expressed as follows:

$$
Q_{v}=\left(\frac{\lambda_{v} a_{v} Q_{\mathrm{N}, v}^{b_{v}}}{C_{v} b_{v}}\right)^{\frac{1}{b_{v}-1}}
$$

Combined with Equation (6), the output energy limit constraint can be rewritten as below:

$$
Q_{v}=\left\{\begin{array}{cc}
Q_{v, \text { min }} & \left(\frac{\lambda_{v} a_{v} Q_{\mathrm{N}, v}^{b_{v}}}{C_{v} b_{v}}\right)^{\frac{1}{b_{v}-1}} \leq Q_{v, \text { min }} \\
\left(\frac{\lambda_{v} a_{v} Q_{\mathrm{N}, v}^{b_{v}}}{C_{v} b_{v}}\right)^{\frac{1}{b_{v}-1}} & Q_{v, \text { min }}<\left(\frac{\lambda_{v} a_{v} Q_{\mathrm{N}, v}^{b_{v}}}{C_{v} b_{v}}\right)^{\frac{1}{b_{v}-1}}<Q_{v, \text { max }} \\
Q_{v, \text { max }} & \left(\frac{\lambda_{v} a_{v} Q_{\mathrm{N}, v}^{b_{v}}}{C_{v} b_{v}}\right)^{\frac{b_{v}-1}{b_{v}}} \geq Q_{v, \text { max }}
\end{array}\right.
$$

When the incremental cost reaches the same value, the total cost is at its optimal value. The specific process is described in detail in Chen et al. [18] and will not be repeated here.

The distributed control strategy seeks to minimize the total life loss cost. However, the following energy allocation methods for the EVs' batteries can also be adopted without considering the total life loss cost:

1) The average allocation method: the required energy is equally allocated into each MESU and the output energy that each MESU needs to offer can be obtained as follows:

$$
Q_{v}=\frac{Q_{\mathrm{D}}}{n}
$$

where $Q_{\mathrm{D}}$ is the amount of energy required by the system.

2) The battery-capacity-based allocation method: the required energy is allocated according to the proportional relationship of the battery's capacity. $Q_{v}$ can be calculated by:

$$
Q_{v}=\frac{Q_{\mathrm{N}, v}}{\sum_{w=1}^{n} Q_{\mathrm{N}, w}} \cdot Q_{\mathrm{D}}
$$

where $\sum_{w=1}^{n} Q_{\mathrm{N}, w}$ is the sum of the batteries' capacity of all EVs.

While adopting the average allocation method or the battery-capacity-based allocation method, $\lambda_{v}=Q_{\mathrm{D}}$ can be guaranteed, and $Q_{\mathrm{D}}$ is issued through the distributed consensus control strategy. The controller calculates the required output energy according to Equations (8) and (9). 
The coefficients of the life loss cost function of batteries have a certain random distribution characteristic when considering the difference among EVs and user habits. We assumed that the coefficients of the life loss cost function were normally distributed. The relationship between the total life loss cost $F_{\text {agg }}$ and the total output energy $Q_{\mathrm{agg}}=\sum_{v=1}^{n} Q_{v}$ during the release process could be obtained as shown in Figure 2.

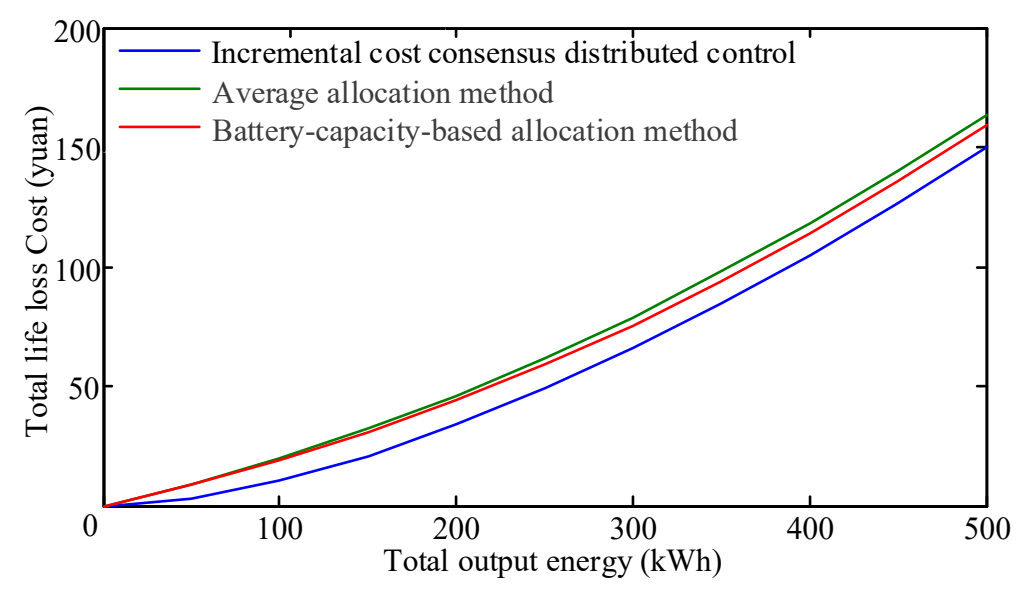

Figure 2. The aggregated models under different allocation methods.

It can be seen from Figure 2 that the total life loss cost and the output energy approximately follow that of a quadratic function. The least squares method was used for fitting the relationship between the life loss cost and the output energy of the cluster in order to establish the cost function model of the MESC. The fitting function is shown as follows:

$$
F_{\text {agg }}=\alpha Q_{\text {agg }}^{2}+\beta Q_{\text {agg }}+\gamma
$$

where $\alpha, \beta$ and $\gamma$ represent the coefficients of the above quadratic function. The cost function curve of the MESC will serve as the basis for implementing the distributed control strategy in the upper layer.

\subsection{Upper Layer Control Strategy}

In order to apply the multi-agent consensus algorithm to the MESS, the reasonable allocation of energy between BESCs is coordinated and the minimum total cost of BESS is guaranteed. The definition of the incremental cost $\lambda_{\mathrm{agg}, i}$ of cluster $i$ is represented as follows:

$$
I_{\mathrm{agg}, i}=\frac{\partial F_{\mathrm{agg}, i}\left(Q_{\mathrm{agg}, i}\right)}{\partial Q_{\mathrm{agg}, i}}=\lambda_{\mathrm{agg}, i}
$$

where $i$ is the index of the MESC, $F_{\mathrm{agg}, i}$ denotes the total life loss cost of cluster $i, Q_{\mathrm{agg}, i}$ is the amount of output energy released by cluster $i$ and $I_{\mathrm{agg}, i}$ is equal to the incremental cost $\lambda_{\mathrm{agg}, i}$ during the operation of the consensus algorithm.

The incremental cost consensus algorithm aims to minimize the total cost of the system while the state of all nodes tends to be consistent. The consensus variable $\lambda_{\mathrm{agg}, i}$ can be obtained by using Equations (10) and (11), which is shown as follows:

$$
\lambda_{\mathrm{agg}, i}=2 \alpha_{i} Q_{\mathrm{agg}, i}+\beta_{i}
$$


The non-leader node interacts with each other following Equation (13):

$$
\lambda_{\mathrm{agg}, i}[k+1]=\sum_{j=1}^{N_{\text {agg }}} h_{i j} \lambda_{\text {agg }, j}[k]
$$

where $N_{\text {agg }}$ represents the total number of clusters, $\lambda_{\mathrm{agg}, j}$ represents the information state of cluster $j$ that is adjacent to cluster $i, h_{i j}$ is the element in the sparse iterative matrix $H$ and the sparse iterative matrix is obtained from the Laplacian matrix corresponding to the communication topology [18].

As seen by Equation (13), the current energy information of each battery can be obtained using the incremental cost, $Q_{\mathrm{agg}, i}$, which can be calculated by:

$$
Q_{\mathrm{agg}, i}=\frac{\lambda_{\mathrm{agg}, i}-\beta_{i}}{2 \alpha_{i}}
$$

Additionally, $\Delta Q$ is defined to indicate the mismatch between the total energy demand of MESS and the overall output energy of all the clusters in order to satisfy the energy balance constraint of the clusters, which is expressed as below:

$$
\begin{gathered}
\Delta Q=Q_{\text {Dagg }}-\sum_{i=1}^{N_{a g g}} Q_{a g g, i} \\
Q_{\text {aggmin }, i} \leq Q_{\text {agg }, i} \leq Q_{\text {aggmax }, i}
\end{gathered}
$$

where $Q_{\text {Dagg }}$ represents the total energy demand of MESS and $Q_{\text {aggmin }, i}$ and $Q_{\text {aggmax }, i}$ are the maximum and minimum output energy, respectively, that each cluster can provide.

The updated rule for the leader node is shown as follows:

$$
\lambda_{\mathrm{agg}, i}[k+1]=\sum_{j=1}^{N_{a g g}} h_{i j} \lambda_{a g g, j}[k]+\varepsilon \Delta Q
$$

where $\varepsilon$ is the convergence coefficient and is related to the convergence speed of the leader node. The convergence rate of the consensus algorithm is related to the topology of the system communication network. $\Delta Q$ is a sign that $\lambda$ increases or decreases. If $\Delta Q>0$, it means that more energy needs to be offered by the energy storage system and the current $\lambda_{\text {agg }}$ should be increased, and vice versa.

In the consensus iteration process, $Q_{\mathrm{agg}, i}$ is subject to the following constraint:

$$
Q_{\mathrm{agg}, i}= \begin{cases}Q_{\mathrm{aggmin}, i} & \frac{\lambda_{\mathrm{agg}, i}-\beta_{i}}{2 \alpha_{i}} \leq Q_{\mathrm{aggmin}, i} \\ \frac{\lambda_{\mathrm{agg}, i}-\beta_{i}}{2 \alpha_{i}} & Q_{\mathrm{aggmin}, i} \leq \frac{\lambda_{\mathrm{agg}, i}-\beta_{i}}{2 \alpha_{i}} \leq Q_{\mathrm{aggmax}, i} \\ Q_{\mathrm{aggmax}, i} & \frac{\lambda_{\mathrm{agg}, i}-\beta_{i}}{2 \alpha_{i}} \geq Q_{\mathrm{aggmax}, i}\end{cases}
$$

By following the updated rules described by Equations (13)-(18), $\lambda_{\mathrm{agg}, i}$ will asymptotically converge to $I_{\text {agg }}$.

The flow chart of the hierarchical distributed collaborative control strategy is shown in Figure 3. When the scheduling command is issued, the control center sends the energy information to the leader node in the upper layer, and the MESCs can realize the optimal allocation by running the consensus algorithm. After energy allocation is completed in the upper layer, each cluster then sends the energy information obtained by itself to the leader node of the lower layer to allocate the energy among EVs. In each iterative process of the consensus algorithm, the non-leader node only needs to apply the consensus algorithm and guarantee the constraint condition. However, in addition to implementing the above process, the leader node also needs to determine whether the balance condition of Equation (15) is satisfied. If the total energy offered by the MESS is equal to the amount of 
energy demanded $(|\Delta Q|<\mu)$, the consensus algorithm is finished and each MESU releases energy according to the information currently obtained by itself, otherwise the consensus algorithm continues to run until the conditions are satisfied.

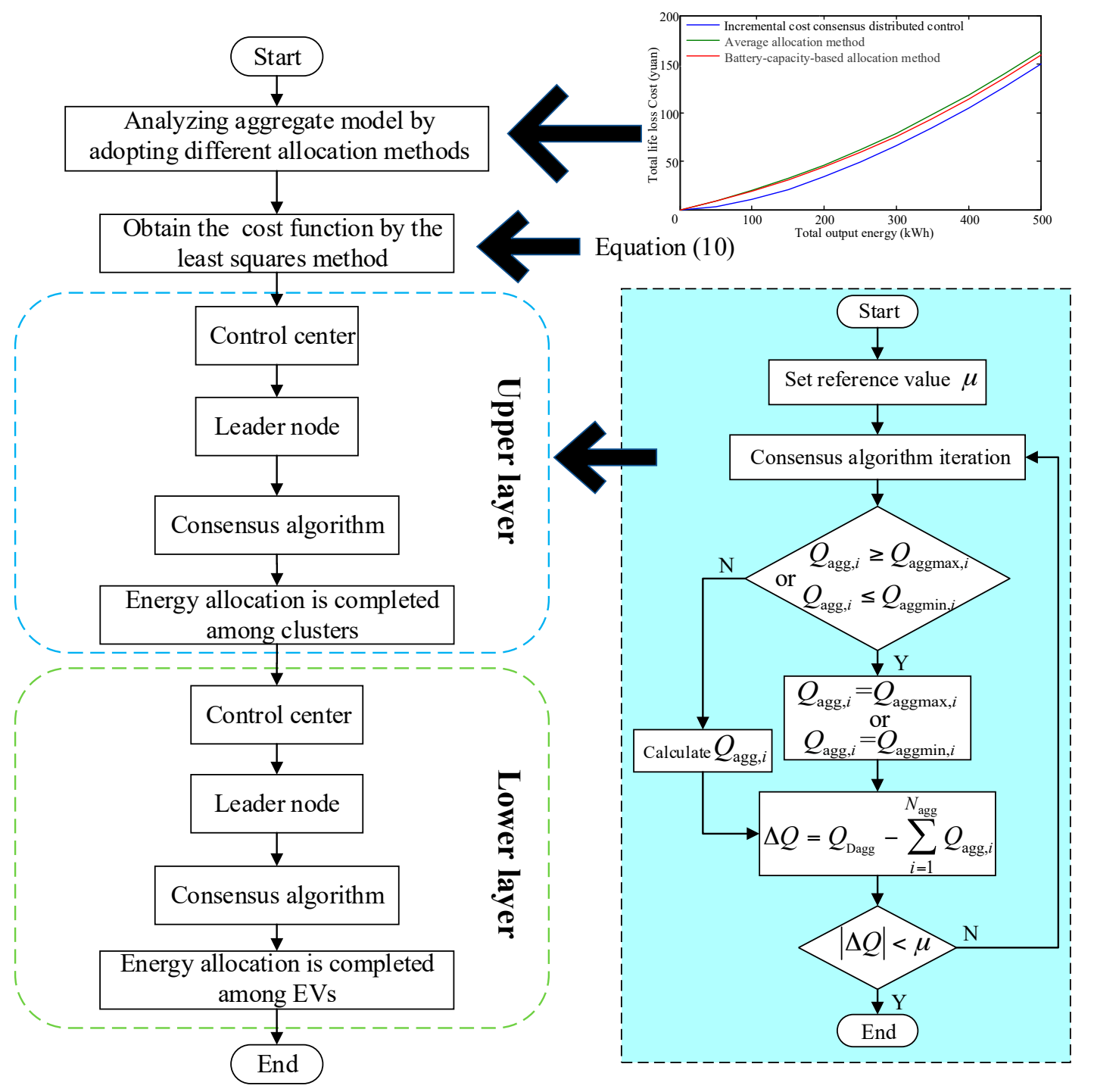

Figure 3. Flowchart of the hierarchical distributed control strategy.

\section{Simulation Results}

Based on the FREEDM microgrid system [19], this paper constructed a distributed energy storage system as shown in Figure 4, and validated the proposed distributed consensus control strategy. MESCs were separately connected to bus I, II, III, IV and V. The dotted line in Figure 4 indicates the communication connection between the clusters. The types and quantities of EVs in each cluster are shown in Table 1.

Table 1. The number and type of electric vehicles (EVs) connected to different buses.

\begin{tabular}{cccc}
\hline Bus & The Number of EVs & BYDe6 & Tengshi \\
\hline I & 32 & 21 & 11 \\
II & 44 & 24 & 20 \\
III & 28 & 15 & 13 \\
IV & 40 & 28 & 12 \\
V & 34 & 19 & 15 \\
\hline
\end{tabular}




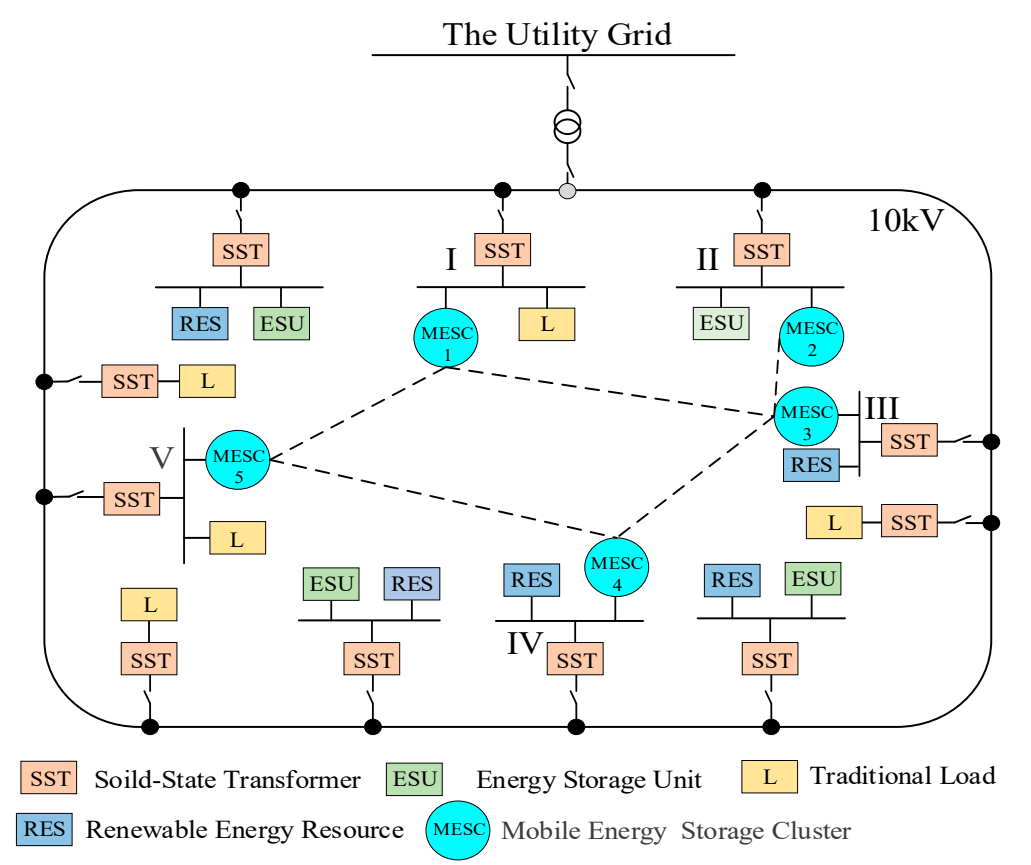

Figure 4. Block diagram of the FREEDM microgrid system.

By using the consensus algorithm and selecting the incremental cost of the cluster as the consensus variable, the dispatch problem can be solved in a distributed manner. Figure 5 shows the communication topology of the five-MESCs system, in which node $1 \sim 5$ corresponded to cluster 1 5 in Figure 4. The incremental cost $\lambda_{\mathrm{agg}, i}$ of cluster $i$ will be updated based on its neighbors' incremental costs. In addition, the leader node has to be selected, which will control whether to increase or decrease the group incremental costs. In the example shown in Figure 5, node 3 was selected as the leader node of the five-MESCs system according to the centrality principle [20].

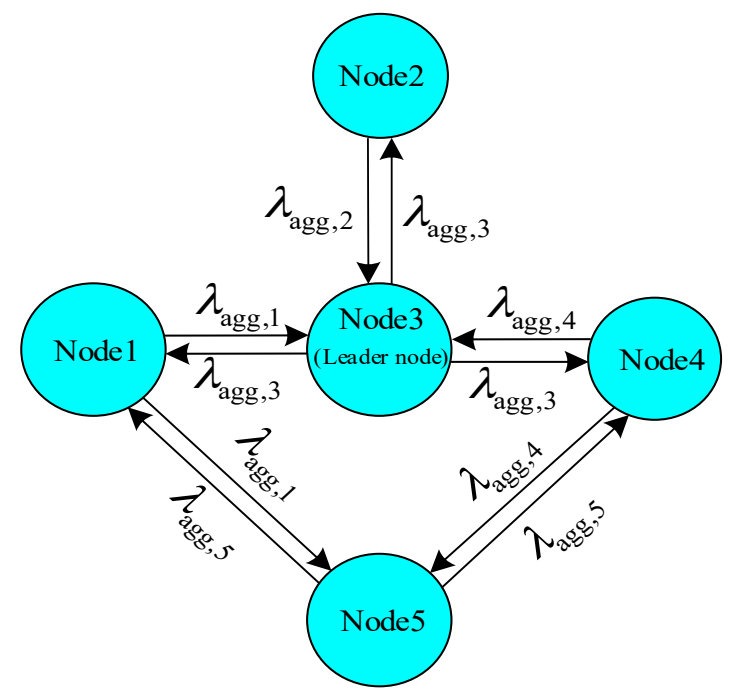

Figure 5. Communication topology of the five mobile energy storage clusters (MESCs) system.

An assumed topology of the EV charging piles in each node is shown in Figure 6, where the dotted line indicates the communication connection and each charging pile can exchange information with four adjacent charging piles (two left and two right). BYDe6 and Tengshi 2017 EVs use Li-ion batteries as energy storage batteries, and their capacities are $82 \mathrm{kWh}$ and $62 \mathrm{kWh}$, respectively. The initial investment cost of the battery is 1300 yuan $/ \mathrm{kWh}$. 


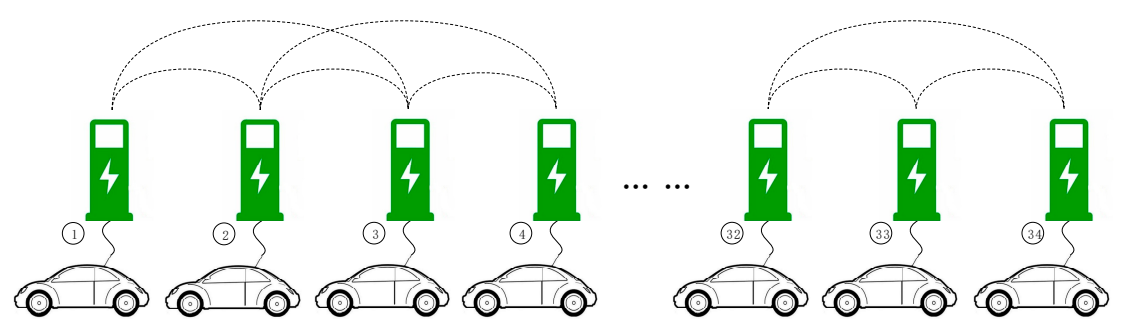

Figure 6. Topology of the EV charging piles.

The above information was used to construct an aggregated model. Methods for allocating output energy of EVs adopt three allocation methods: the optimal allocation method based on the consensus incremental cost, the average allocation method and the battery-capacity-based allocation method. The coefficients of the function of the aggregated model are shown in Tables $2-4$ for each allocation method, respectively.

Table 2. The parameters of the five mobile energy storage clusters (MESCs) system using the optimal allocation method based on the consensus incremental cost.

\begin{tabular}{cccc}
\hline Node & $\boldsymbol{\alpha}$ & $\boldsymbol{\beta}$ & $\boldsymbol{\gamma}$ \\
\hline 1 & 0.0004 & 0.0849 & -1.3760 \\
2 & 0.0003 & 0.0715 & -1.3610 \\
3 & 0.0005 & 0.0738 & -1.3071 \\
4 & 0.0004 & 0.0737 & -1.5293 \\
5 & 0.0004 & 0.1030 & -1.6182 \\
\hline
\end{tabular}

Table 3. The parameters of the five-MESCs system using the average allocation method.

\begin{tabular}{cccc}
\hline Node & $\boldsymbol{\alpha}$ & $\boldsymbol{\beta}$ & $\gamma$ \\
\hline 1 & 0.0003 & 0.1402 & 0.1734 \\
2 & 0.0002 & 0.1503 & 0.0632 \\
3 & 0.0004 & 0.1484 & 0.1242 \\
4 & 0.0003 & 0.1752 & 0.0624 \\
5 & 0.0003 & 0.1645 & 0.0528 \\
\hline
\end{tabular}

Table 4. The parameters of the five-MESCs system using the battery-capacity-based allocation method.

\begin{tabular}{cccc}
\hline Node & $\boldsymbol{\alpha}$ & $\boldsymbol{\beta}$ & $\boldsymbol{\gamma}$ \\
\hline 1 & 0.0003 & 0.1276 & 0.2084 \\
2 & 0.0002 & 0.1414 & 0.0728 \\
3 & 0.0004 & 0.1379 & 0.1616 \\
4 & 0.0003 & 0.1598 & 0.0693 \\
5 & 0.0003 & 0.1531 & 0.0423 \\
\hline
\end{tabular}

Assuming that a lack of energy occurred at $t=0 \mathrm{~s}$, a scheduling command was issued, which was required to supply energy about $500 \mathrm{kWh}$ to the system in a short time.

Firstly, the hierarchical distributed cooperative control strategy proposed in this paper was tested. After receiving the scheduling command, the five-MESCs system realized the optimal allocation of the output energy in the upper layer through the distributed control. Then, after the nodes in the lower layer received the energy information, the output energy was allocated by the corresponding allocation strategy. A fixed step size of $0.02 \mathrm{~s}$ and a convergence coefficient of $\varepsilon=0.00005$ were used. It is worth mentioning that the aggregated model of the MESC was modeled based on the three allocation methods given in Section 3.1: the optimal allocation method based on consensus incremental cost, the average allocation method and the battery-capacity-based allocation method. The related simulation results are shown in Figures 7 and 8. 

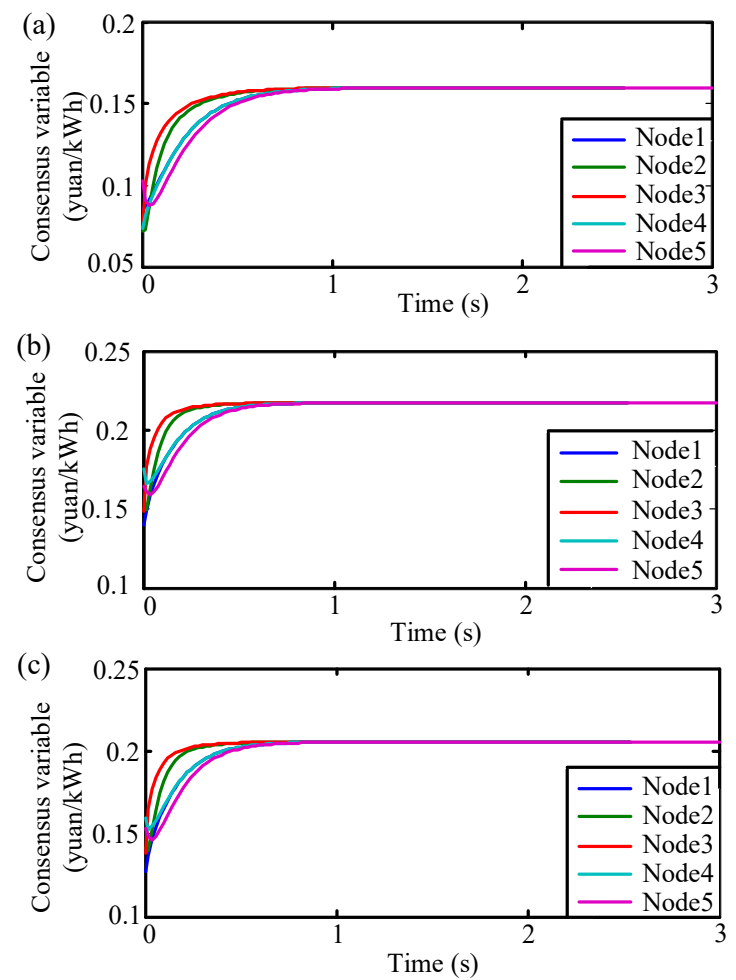

Figure 7. Consensus variables $\lambda_{\mathrm{agg}, j}$ in the upper layer for (a) the optimal allocation method based on the consensus incremental cost, (b) the average allocation method and (c) the battery-capacity-based allocation method.
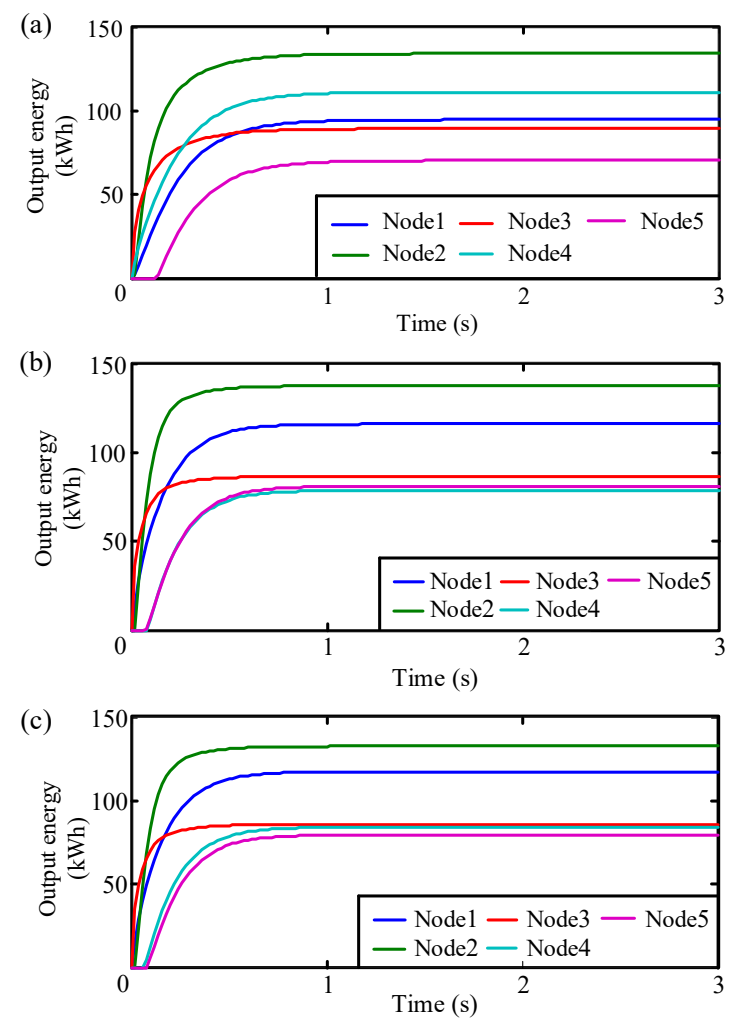

Figure 8. Output energy $Q_{\mathrm{agg}, i}$ in the upper layer for (a) the optimal allocation method based on the consensus incremental cost, (b) the average allocation method and (c) the battery-capacity-based allocation method. 
Figures 7 and 8 indicate that the system reached a steady state in a short time. At the same time, the energy storage clusters could realize the optimal energy allocation according to the distributed control strategy. No matter which method was used, energy can be efficiently allocated to each node in the upper layer with its optimal cost.

The information of each node under different energy allocation methods is shown in Table 5.

Table 5. The energy information of each node under different allocation methods.

\begin{tabular}{cccc}
\hline Node & $\begin{array}{c}\text { Optimal Allocation Based on the } \\
\text { Consensus Incremental Cost (kWh) }\end{array}$ & Average Allocation (kWh) & $\begin{array}{c}\text { Battery-Capacity-Based } \\
\text { Allocation (kWh) }\end{array}$ \\
\hline 1 & 94.7762 & 116.1089 & 117.6006 \\
2 & 134.2594 & 137.6080 & 132.6827 \\
3 & 89.3234 & 86.5212 & 85.9543 \\
4 & 111.3019 & 78.7278 & 84.2197 \\
5 & 70.3390 & 81.0341 & 79.5427 \\
\hline
\end{tabular}

It can be observed in Table 5 that the batteries that adopted different allocation methods in the lower layer led to a different output energy in the upper layer. However, the total energy demanded by the system was unchanged, so the total output energy was equal to the energy required by the system. Eventually, the amount of output energy scheduled by each EV could be obtained by calculations.

The life loss cost under different allocation methods are shown in Table 6. As shown in Table 6, the overall life loss cost of the five-MESCs system was at its minimal when adopting the optimal allocation based on the consensus incremental cost method.

Table 6. The life loss cost of batteries under different allocation methods.

\begin{tabular}{cc}
\hline Allocation Method & Cost (yuan) \\
\hline Optimal allocation based on the consensus of & 52.6900 \\
incremental cost & 93.2611 \\
Average allocation & 87.5728 \\
\hline Battery-capacity-based allocation & \\
\hline
\end{tabular}

After the energy allocation in the upper layer was completed, the energy allocation among EVs in the lower layer was performed. For example in cluster 4, the corresponding output energy was $111.3019 \mathrm{kWh}$ and that corresponds to $Q_{\mathrm{D}}=111.3019 \mathrm{kWh}$. The simulation curves are shown in Figures 9 and 10 using the communication topology in Figure 6.

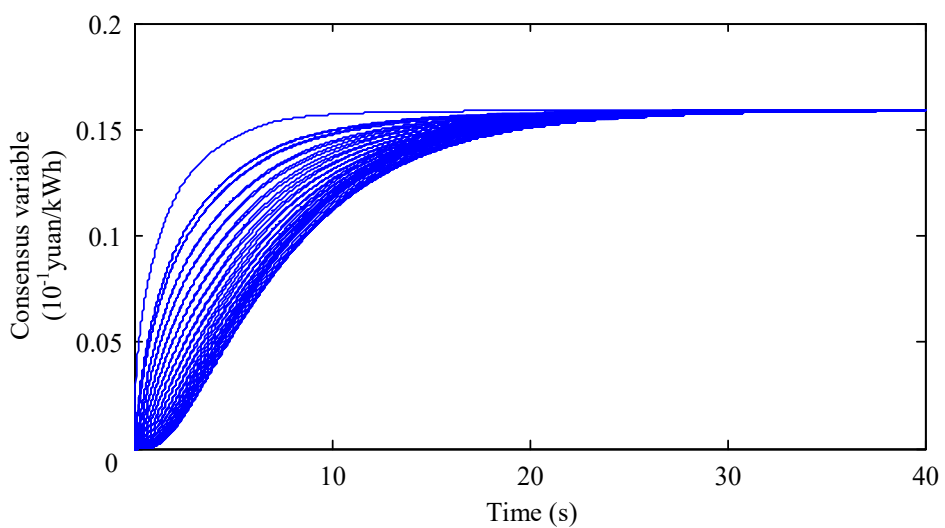

Figure 9. Consensus variables $\lambda_{v}$ in cluster 4 . 


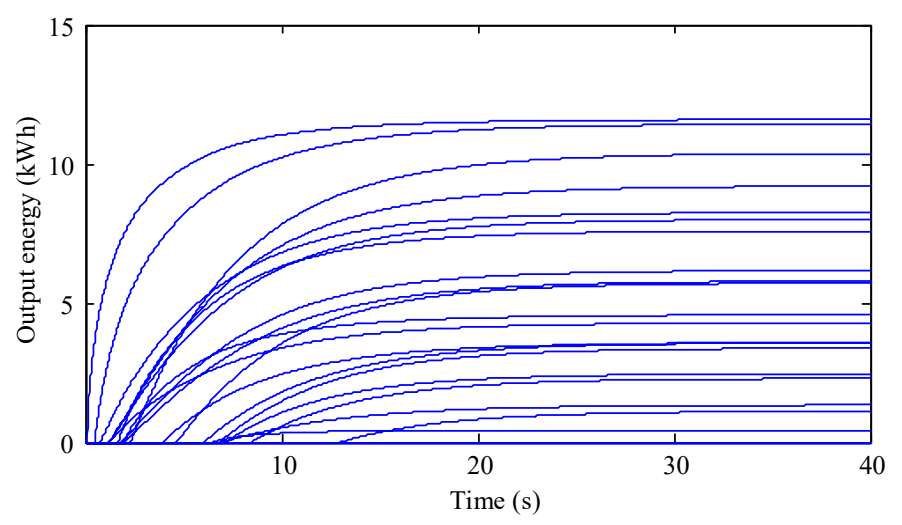

Figure 10. Output energy $Q_{v}$ in cluster 4.

In the above example, each EV charging pile only exchanged information with four adjacent charging piles. If the information interaction capability changes, it will affect the communication topology and the final response time. With the enhancement of the wireless communication capability, each charging pile can exchange information with more adjacent charging piles based on the charging piles distribution structure shown in Figure 6, which leads to a change in the communication topology. The relationship between response time, consensus variable and output energy under different communication capabilities is shown in Figure 11, where the number of charging piles that can exchange information reflected the communication capability.

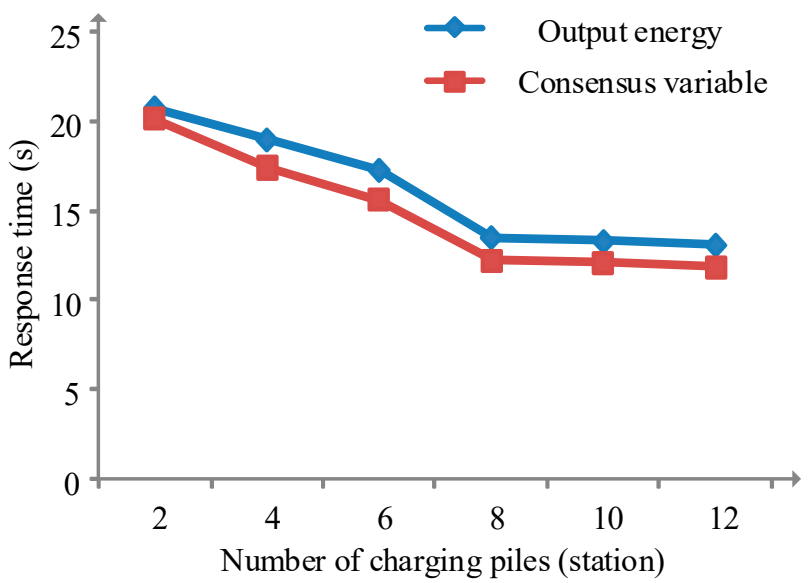

Figure 11. Response time under different communication capabilities.

As shown in Figure 11, the system converged to the target value more quickly and responded more quickly to the scheduling command with the enhancement of the wireless signal communication capability. However, when the number of charging piles were more than eight, there was no significant change in the response time with increasing number of charging piles.

In order to illustrate the superiority of the hierarchical control, the following examples adopted the non-hierarchical control method to allocate energy to all EVs. Compared with the hierarchical distributed control method, all EVs directly participated in energy allocation, which made the structure of the system more complicated, resulting in a slower convergence of the consensus algorithm in the iterative process and a longer time to reach optimal results. The simulation curves are shown in Figures 12 and 13. 


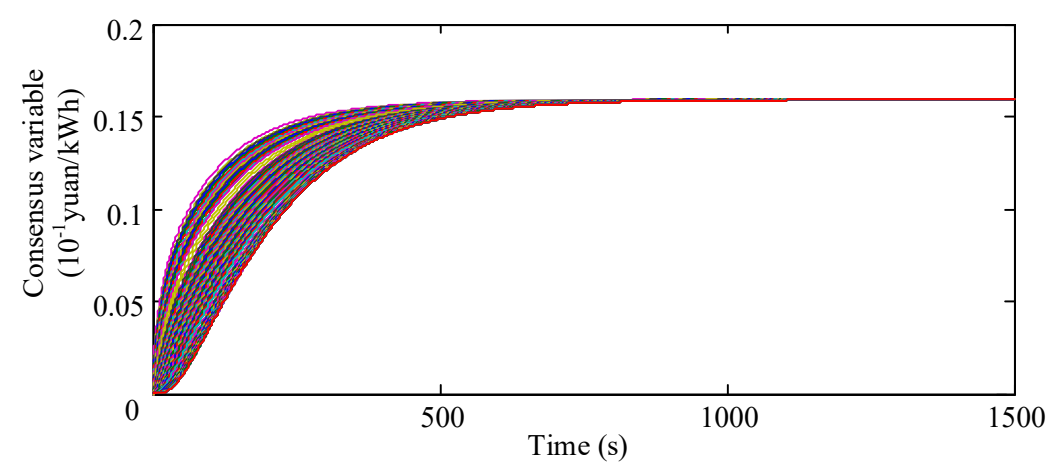

Figure 12. Curves of the consensus variable of each EV under the non-hierarchical control.

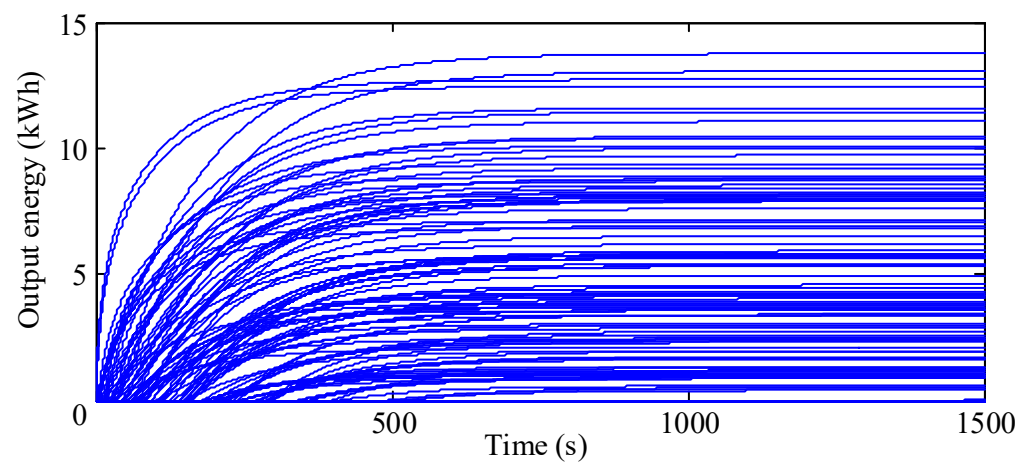

Figure 13. Curves of the output energy of each EV under the non-hierarchical control.

Figures 12 and 13 show the curves of the consensus variables and output energy of all EVs in the system while adopting the non-hierarchical control method. As shown in Figure 12, the time taken for the consensus variable to converge to the optimal value reached $800 \mathrm{~s}$ when the non-hierarchical control was adopted. According to the curves of the output energy shown in Figure 13, the system scheduling cost was about 47.56 yuan. Although the overall cost was slightly reduced, the total response time under the hierarchical distributed control method (Figures 7 and 8 and Table 6) was less than $5 \%$ of that when the control was non-hierarchical.

\section{Conclusions}

This paper proposed a hierarchical distributed collaborative control strategy for MESCs. Firstly, the aggregate characteristics of EVs with different energy allocation methods adopted were analyzed. According to the relationship between the scheduling cost and output energy, the function of the aggregated model in the lower layer was constructed by the least squares method. The distributed control strategy in the upper layer was then proposed with the aggregated model. In terms of simulation, this paper started with the topology structure of theMESC, and analyzed the characteristics of the distributed consensus algorithm in the convergence of the results and the time required. The simulation results implied that the hierarchical distributed control strategy proposed in this paper could not only realize the allocation of energy demanded in the system, but also optimize the total scheduling cost and shorten the response time.

Author Contributions: All authors contributed equally to the work.

Acknowledgments: This work was supported by National Natural Science Foundation of China (No. 51707099), the University Science Research Project of Jiangsu Province (No. 16KJB470009), and the China Postdoctoral Science Foundation (No. 2017M611859).

Conflicts of Interest: The authors declare no conflicts of interest. 


\section{References}

1. Cecati, C.; Citro, C.; Siano, P. Combined Operations of Renewable Energy Systems and Responsive Demand in a Smart Grid. IEEE Trans. Sustain. Energy 2011, 2, 468-476. [CrossRef]

2. Li, B.; Xu, T.; Fang, Y.; Xue, F.; Sheng, X. On-line security assessment and control decision support for large amount wind power integrated power system. Int. J. Electr. Power Energy Syst. 2016, 83, 15-20.

3. Moens, L.; Blake, D.M.; Rudnicki, D.L.; Hale, M.J. Advanced thermal storage fluids for solar parabolic trough systems. J. Sol. Energy-Trans. ASME 2003, 125, 112-116. [CrossRef]

4. Li, P. Energy storage is the core of renewable technologies. IEEE Nanotechnol. Mag. 2008, 2, 13-18. [CrossRef]

5. Wu, D.; Aliprantis, D.C.; Ying, L. Load Scheduling and Dispatch for Aggregators of Plug-In Electric Vehicles. IEEE Trans. Smart Grid 2012, 3, 368-376. [CrossRef]

6. Radu, A.T.; Eremia, M.; Toma, L. Promoting battery energy storage systems to support electric vehicle charging strategies in smart grids. In Proceedings of the Electric Vehicles International Conference, Bucharest, Romania, 5-6 October 2017.

7. Cao, Y.J.; Tang, S.W.; Li, C.B.; Zhang, P.; Tan, Y.; Zhang, Z.K.; Li, J.X. An Optimized EV Charging Model Considering TOU Price and SOC Curve. IEEE Trans. Smart Grid 2012, 3, 388-393. [CrossRef]

8. Hui, C.; Chen, Q.; Guan, Z.; Huang, J.J.P.; Systems, C.o.M.P. Day-ahead optimal charging/discharging scheduling for electric vehicles in microgrids. Prot. Control Mod. Power Syst. 2018, 3, 9.

9. Han, S.; Han, S.; Sezaki, K. Development of an Optimal Vehicle-to-Grid Aggregator for Frequency Regulation. IEEE Trans. Smart Grid 2010, 1, 65-72. [CrossRef]

10. Ping, Y.; Zhu, T.; Lin, G.; Zhang, Q. Routing Renewable Energy Using Electric Vehicles in Mobile Electrical Grid. In Proceedings of the IEEE International Conference on Mobile Ad-hoc \& Sensor Systems, Hangzhou, China, 14-16 October 2013.

11. Kong, F.X.; Liu, X. Distributed Deadline and Renewable Aware Electric Vehicle Demand Response in the Smart Grid. In Proceedings of the 2015 IEEE Real-Time Systems Symposium, San Antonio, TX, USA, 1-4 December 2015; pp. 23-32. [CrossRef]

12. Wu, X.; Wang, X.; Li, J.; Guo, J.; Hang, K.; Chen, J. A Joint Operation Model and Solution for Hybrid Wind Energy Storage Systems. Proc. CSEE 2013, 33, 10-17.

13. Lü, C. Real time economic scheduling method for photovoltaic/battery system based on the evaluation of energy storage charging cost and discharge yield. Power Syst. Prot. Control 2017, 45, 144-151. [CrossRef]

14. Hong, H.; Jiang, Q.; Yan, Y. An Optimization Control Method of Battery Energy Storage System with Wind Power Fluctuations Smoothed in Real Time. Autom. Electr. Power Syst. 2013, 37, 103-109. [CrossRef]

15. Dominguez-Garcia, A.D.; Cady, S.T.; Hadjicostis, C.N. Decentralized Optimal Dispatch of Distributed Energy Resources. In Proceedings of the 2012 IEEE 51st IEEE Conference on Decision and Control (CDC), Maui, HI, USA, 10-13 December 2012; pp. 3688-3693.

16. Zhang, Y.; Rahbari-Asr, N.; Duan, J.; Chow, M.Y. Day-Ahead Smart Grid Cooperative Distributed Energy Scheduling With Renewable and Storage Integration. IEEE Trans. Sustain. Energy 2016, 7, 1739-1748. [CrossRef]

17. Khazaei, J.; Miao, Z. Consensus Control for Energy Storage Systems. IEEE Trans. Smart Grid 2016, PP, 1.

18. Chen, G.; Bao, Y.-Q.; Zhang, J.; Wang, B.; Wu, M.; Wang, T. Distributed Cooperative Control Strategy for Energy Storage Units Considering Life Loss Cost. Power Syst. Technol. 2018, 42, 1495-1501.

19. Zhang, M.R.; Chen, J. The Energy Management and Optimized Operation of Electric Vehicles Based on Microgrid. IEEE Trans. Power Deliv. 2014, 29, 1427-1435. [CrossRef]

20. Kar, S.; Hug, G. Distributed Robust Economic Dispatch in Power Systems: A Consensus plus Innovations Approach. In Proceedings of the 2012 IEEE Power and Energy Society General Meeting, San Diego, CA, USA, 22-26 July 2012.

(C) 2019 by the authors. Licensee MDPI, Basel, Switzerland. This article is an open access article distributed under the terms and conditions of the Creative Commons Attribution (CC BY) license (http:/ / creativecommons.org/licenses/by/4.0/). 